by Vincent R. Lalli

Lewis Research Center

Cleveland, Ohio

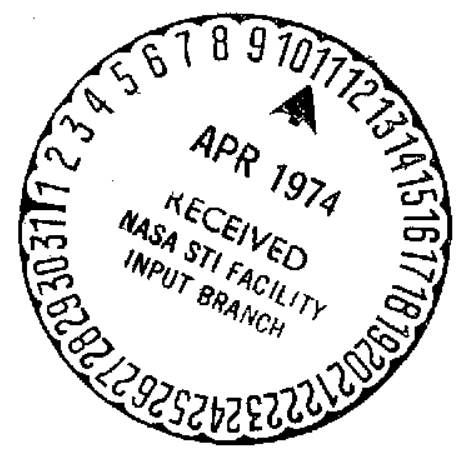

TECHNICAL PAPER proposed for presentation at

Semiconductor Electronics Conference sponsored by the Institute of Electrical and Electronic Engineers

Cleveland, Ohio, May 9, 1974 


\title{
1 \\ CONVERTER DESIGN TECHNIQUES AND APPLICATIONS
}

by Vincent $R$. Lalli

\begin{abstract}
Transistorized direct current voltage converters offer a number of advantages over other types because of their small size, low weight, high efficiency, high reliability, precision accuracy and wide range of control,

There are three basic types of converter subsystems: series converter in which a voltage-controlled component is placed in series with the load; shunt converter in which a current-controlled component is placed in shunt with the load; and a switching converter in which a voltage-controlled component is turned on and off in series with the load. Converters employing any of these three types of subsystems can provide constant voltage, constant current, or constant impedance across the load.

This paper discusses transistorized voltage converters of the series, shunt, and switching types. Included are design and application considerations, step-by-step design procedures, and the solution to sample design problems.
\end{abstract}




\section{INTRODUCTION}

The function of power converters in aerospace systems is to transfer and transform electric power from the output terminals of a power source (i.e., generator) to other forms as required by specific loads. Efficiency, size, weight, reliability, ripple, regulation, electromagnetic compatibility and stability are important parameters in aerospace power processing systems. The design and application considerations are discussed herein for transistorized voltage converters of the series, shunt, and switching configurations. Power processing systems are made up of power converter subsystems (ref. 1).

A typical series voltage converter is shown in figure 1. In this converter, output voltage $\left(V_{0}\right)$ is sensed by a resistor network (R2 and R3). The analog voltage $\left(V_{3}\right)$ is compared to a reference voltage $\left(V_{R}\right)$ to generate an error signal $\left(\mathrm{V}_{\mathrm{E}}\right)$ to drive the series component. Bias for the series component is obtained from the generator $\left(V_{G}\right)$ thru resistor (R1). The load resistor $\left(R_{0}\right)$ varies over the range from $\left(R_{0}-\Delta R_{0}\right)$ to $\left(R_{0}+\Delta R_{0}\right)_{0}$ The voltage source $\left(V_{s}\right)$ varies over the range from $\left(V_{S}-\Delta V_{S}\right)$ to $\left(V_{S}+\Delta V_{S}\right)$ with a generator resistance $\left(R_{G}\right)$. The voltage appearing at the generator terminals is $\left(\mathrm{V}_{\mathrm{G}}\right)$.

A typical shunt voltage converter is shown in figure 2. The shunt regulator includes a shunt component and a reference-voltage component. The output-voltage $\left(\mathrm{V}_{\mathrm{o}}\right)$ remains constant because the shunt-element current $\left(\mathrm{I}_{\mathrm{Sh}}\right)$ changes as the load current $\left(\mathrm{I}_{\mathrm{o}}\right)$ or input voltage $\left(\mathrm{V}_{\mathrm{s}}\right)$ changes. The shunt element current $\left(\mathrm{I}_{\mathrm{Sh}}\right)$ change is reflected in a change of voltage across the resistor (R1) in series with the load.

A typical switching voltage converter is shown in figure 3 . The series component serves as a switch and is either shut off or full on (saturated). When the current thru the series component is high, the voltage drop across it is low, so the power dissipated in the series component is minimized. The output voltage is sampled and compared to a reference. The error signal is amplified in the voltage comparator. 
The amplified error signal drives the interface component to turn the series component on or off depending on the source or load activity. When the series component is on, the free wheeling diode $\left(D_{F}\right)$ is backbiased by the generator voltage $\left(\mathrm{V}_{\mathrm{G}}\right)$. During saturation of the series component the voltage across the filter choke $\left(V_{F}\right)$ is the difference between the generator voltage $\left(\mathrm{V}_{\mathrm{G}}\right)$ and the output voltage $\left(\mathrm{V}_{\mathrm{O}}\right)$ which is nearly a constant. Since the voltage across the choke during this time is nearly constant, the current through the choke increases linearly with time. When the output voltage reaches a predetermined level, the voltage comparator turns the series component off. When the series component is off, the current in the choke starts to decrease, so the voltage across the choke reverses polarity. This polarity change is in the direction to turn on the filter diode $\left(D_{F}\right)$, and the choke then becomes clamped to the filter capacitor $\left(\mathrm{C}_{\mathrm{F}}\right)$, which has the output voltage $\left(\mathrm{V}_{\mathrm{o}}\right)$ across it. The choke current then decreases linearly during the off time interval. When the choke current falls below the load current, the capacitor supplies the difference to the load. This causes the capacitor voltage to fall. When the output voltage falls below a level determined by the voltage reference, the series component is turned back on. The on/off ratio increases with load and decreasing generator voltage.

\section{SERIES CONVERTER DESIGN TECHNIQUE}

The step-by-step technique which follows is recommended for the design of transistorized series voltage converters. The design equations used in this technique are derived in Ref. 2. Referring to the block diagram in figure 1

1. Conditions and requirements typical for a spacecraft application:

$$
\begin{array}{ll}
\mathrm{V}_{\mathrm{O}}=28 \mathrm{Vdc} & \Delta \mathrm{V}_{\mathrm{O}}= \pm 0.1 \mathrm{Vdc} \\
\mathrm{V}_{\mathrm{S}}=56 \mathrm{Vdc} & \Delta \mathrm{V}_{\mathrm{S}}= \pm 6 \mathrm{Vdc} ; \text { solar array battery buffered. }
\end{array}
$$


$R_{\mathrm{G}}=7 \mathrm{ohms}$

$\mathrm{R}_{\mathrm{O}}=40$ ohms $\quad \Delta \mathrm{R}_{\mathrm{o}}= \pm 15 \mathrm{ohms}$

Spacecraft baseplate temperature $-55^{\circ}$ to $+60^{\circ} \mathrm{C}$

2. Select a silicon reference diode

$$
\begin{aligned}
& \text { Usually } 0.2 \mathrm{~V}_{\mathrm{o}} \leq \mathrm{V}_{\mathrm{R}} \leq 0.9 \mathrm{~V}_{\mathrm{o}} ; \text { choose } \\
& \mathrm{V}_{\mathrm{R}} \simeq 0.5 \mathrm{~V}_{\mathrm{o}} \simeq 14 \mathrm{Vdc} \text { use } 15 \mathrm{Vdc}
\end{aligned}
$$

The values of $\mathrm{V}_{\mathrm{R}}, \Delta \mathrm{V}_{\mathrm{o}}$ and circuit gain are all interrelated. Higher values of $V_{R}$ minimize circuit gain for a given $\Delta \mathrm{V}_{\mathrm{O}}$.

In selecting electronic components, a reasonable value of temperature rise $(\Delta \mathrm{T})$ for the components must be determined. Typical values are $15^{\circ} \mathrm{C}$ for $\Delta \mathrm{T}$.

Motorola type (Ref. 3) IN965B IN4109

Power rating at $75^{\circ} \mathrm{C} \quad 368 \quad 140 \mathrm{~mW}$

Current rating at $75^{\circ} \mathrm{C} \quad 24.5 \quad 9.35 \mathrm{~mA}$

$\begin{array}{lll}\text { Cost } & 2.95 & 3.85 \$\end{array}$

Reference supply $\left(\mathbf{I}_{\mathrm{RS}}\right)=10 \mathrm{~mA}$ typical

Power dissipation $\left(\mathrm{PD}_{\mathrm{RC}}\right)=\mathrm{V}_{\mathrm{R}} \times \mathrm{I}_{\mathrm{RS}}=150 \mathrm{~mW}$.

Select IN965B for reference diode

3. Determine $\mathrm{V}_{3}$

$\mathrm{V}_{3}$ is a function of $\mathrm{V}_{\mathrm{R}}$, typically $\mathrm{V}_{3}=\mathrm{V}_{\mathrm{R}}$

$\therefore \mathrm{V}_{3}=15.0 \mathrm{Vdc}$

4. Select a series component

$$
\begin{aligned}
\mathrm{I}_{\mathrm{O}}=0.70 \mathrm{Adc} \quad \Delta \mathrm{I}_{\mathrm{O}}= & +0.42 \\
& -0.19
\end{aligned}
$$




$$
\begin{aligned}
& \mathrm{V}_{\mathrm{CE}_{\max } \text { (steady state) }} \geq \mathrm{V}_{\mathrm{S}}+\Delta \mathrm{V}_{\mathrm{s}}-\mathrm{V}_{\mathrm{o}}\left(1+\frac{\mathrm{R}_{\mathrm{G}}}{\mathrm{R}_{\mathrm{o}}+\Delta \mathrm{R}_{\mathrm{o}}}\right)-\Delta \mathrm{V}_{\mathrm{o}} \geq 56 \\
& +6-28\left(1+\frac{7.0}{55}\right)-0.1 \geq 30.3 \mathrm{Vdc} \\
& \mathrm{V}_{\mathrm{CE} \text { min }} \leq \mathrm{V}_{\mathrm{s}}-\Delta \mathrm{V}_{\mathrm{S}}-\mathrm{V}_{\mathrm{o}}\left(1+\frac{\mathrm{R}_{\mathrm{G}}}{\mathrm{R}_{\mathrm{o}}-\Delta \mathrm{R}_{\mathrm{o}}}\right)+\Delta \mathrm{V}_{\mathrm{o}} \leq 56 \\
& -6-28\left(1+\frac{7}{25}\right)+0,1 \leq 14.2 \mathrm{Vdc} \\
& \mathrm{I}_{\mathrm{C}_{\max }}=\mathrm{I}_{\mathrm{O} \text { max }} \\
& \mathrm{PD}_{\mathrm{T}_{\max }}=\left(\mathrm{V}_{\mathrm{s}}+\Delta \mathrm{V}_{\mathrm{s}}-\mathrm{V}_{\mathrm{o}}-\Delta \mathrm{V}_{\mathrm{o}}\right) \mathrm{I}_{\mathrm{C}_{\max }}-\left(\mathrm{I}_{\mathrm{C}_{\max }}\right)^{2}\left(\mathrm{R}_{\mathrm{G}}\right) \\
& \simeq 34(1.12)-(1.12)^{2}(7)=29.2 \mathrm{Wdc} \text { at a maximum case }
\end{aligned}
$$

temperature of $75^{\circ} \mathrm{C}$. Consider

$\begin{array}{lrr}\text { Motorola type (NPN) } & 2 \mathrm{~N} 4231 & 2 \mathrm{~N} 4233 \\ \mathrm{BV}_{\mathrm{CEO}} & 40 & 80 \mathrm{Vdc} \\ \mathrm{V}_{\mathrm{CE}} & 2.0 & 2.0 \mathrm{Vdc} \\ { }_{\text {sat }}{ }_{\max } & 1.5 & 1.5 \mathrm{Adc} \\ \mathrm{I}_{\mathrm{max}} & 28.0 & 28.0 \mathrm{~W} \\ \mathrm{PD}_{\mathrm{T}} \text { at } 75^{\circ} \mathrm{C} & 50 & 50 \\ \mathrm{~h}_{\mathrm{FE}} \text { at } 0.70 \mathrm{Adc} & 5.0 & 5.0 \mathrm{Vdc} \\ \mathrm{V}_{\mathrm{EB}} & & \end{array}$


$\mathrm{h}_{\mathrm{fe}}$

$\mathrm{g}_{\mathrm{m}}$ at $75^{\circ} \mathrm{C}$

30

30

Cost

4. 0

4. 0 mhos

2.10

$3.20 \$$

Choose the 2N4231

5. Select a Darlington driver

Choose $\mathrm{I}_{\mathrm{Bn}}=1.0 \mathrm{mAdc}=$ input base current of $\mathrm{n}^{\text {th }}$ transistor

$\mathrm{h}_{\mathrm{FE}_{1}}{ }_{1} \mathrm{FE}_{2} \cdots \mathrm{h}_{\mathrm{FE}}=\frac{\mathrm{I}_{\mathrm{C} 1}}{\mathrm{I}_{\mathrm{Bn}}}=\frac{0.70}{1 \times 10^{-3}}=700$

$\mathrm{h}_{\mathrm{FE}_{2}} \geq \frac{\mathrm{I}_{\mathrm{C} 1}}{\mathrm{I}_{\mathrm{Bn}}} \cdot \frac{1}{\mathrm{~h}_{\mathrm{FE}_{1}}}=\frac{700}{50}=14$

$\mathrm{I}_{\mathrm{C} 2}=\mathrm{I}_{\mathrm{B} 1}=\frac{\mathrm{I}_{\mathrm{C} 1}}{\mathrm{~h}_{\mathrm{FE}_{1}}}=\frac{0.70}{50}=15 \mathrm{mAdc}$

$\mathrm{PD}_{\mathrm{T}_{(\max )}} \simeq\left(\mathrm{V}_{\mathrm{CE}_{1}}-\mathrm{V}_{\mathrm{BE}_{1}}\right) \mathrm{I}_{\mathrm{C} 2}=(30.3-0.7) 15 \times 10^{-3}$

$=29.6 \times 15 \times 10^{-3}=445 \mathrm{~mW}$

$\begin{array}{lll}\text { Consider Motorola type (NPN) } & \text { 2N3946 } & \text { 2N3947 }\end{array}$

${ }^{\mathrm{BV}} \mathrm{CEO}_{\max }$

$\mathrm{I}_{\mathrm{max}}$

$40 \quad 40 \mathrm{Vdc}$

$\mathrm{PD}_{\mathrm{T}_{\max }}$ at $75^{\circ} \mathrm{C}$

$200200 \mathrm{mAdc}$

${ }^{\mathrm{FE}}$ at $15 \mathrm{mAdc}$

$0.96 \quad 0.96 \mathrm{~W}$

$\mathrm{V}_{\mathrm{EB}}$

100

150

$6.0 \quad 6.0 \mathrm{Vdc}$ 
$\begin{array}{lrr}\mathrm{g}_{\mathrm{m}} & 0.25 & 0.35 \mathrm{mho} \\ \mathrm{Cost} & 2.40 & 2.65 \$\end{array}$

Choose 2N3946

6. Solve for $\mathrm{V}_{\mathrm{BE}_{21}}$, where

$$
\mathrm{v}_{\mathrm{BE}_{21}}=\mathrm{v}_{\mathrm{BE}_{2}}+\mathrm{v}_{\mathrm{BE}_{1}}=0.77+0.83=1.60 \mathrm{Vdc}
$$

7. The design-center value for $\mathrm{V}_{\mathrm{CE}_{1}}$ is given by

$$
\mathrm{V}_{\mathrm{CE}_{1}}=\mathrm{V}_{\mathrm{S}}-\mathrm{V}_{\mathrm{O}}-\frac{\mathrm{V}_{\mathrm{O}} \mathrm{R}_{\mathrm{G}_{\mathrm{O}}}}{\mathrm{R}_{\mathrm{O}}^{2}-\Delta \mathrm{R}_{\mathrm{O}}^{2}}=56-28-\frac{(28)(7)(40)}{(40)^{2}-(15)^{2}}=22.3 \mathrm{Vdc}
$$

8. Solve for $R_{1}$

$$
\mathrm{R}_{1}=\frac{\mathrm{V}_{\mathrm{CE}_{1}}-\mathrm{V}_{\mathrm{BE}_{21}}}{\mathrm{I}_{\mathrm{B} 2}}=\frac{22.3-1.60}{1 \times 10^{-3}}=20.8 \mathrm{Kohms}
$$

Use $21 \mathrm{~K}$ ohms

9. Solve for $\mathrm{g}_{\mathrm{m} 3}=\frac{\Delta \mathrm{I}_{\mathrm{C}}}{\Delta \mathrm{V}_{\mathrm{BE}}}$; where $\mathrm{g}_{\mathrm{m} 3}$ is the mutual conductance of the amplifier component (transistor).

$$
\Delta \mathrm{V}_{\mathrm{o}}=\frac{\Delta \mathrm{V}_{\mathrm{S}}+\frac{\mathrm{V}_{\mathrm{o}}}{\mathrm{R}_{\mathrm{o}}^{2}}\left(\mathrm{R}_{\mathrm{G}}+\frac{1}{\mathrm{~g}_{\mathrm{m} 1}}\right) \Delta \mathrm{R}_{\mathrm{o}}}{1+\mathrm{g}_{\mathrm{m} 3} \mathrm{R}_{1} \frac{\mathrm{V}_{3}}{\mathrm{~V}_{\mathrm{o}}}+\frac{\mathrm{R}_{\mathrm{G}}}{\mathrm{R}_{\mathrm{o}}}+\frac{1}{\mathrm{R}_{\mathrm{o}} \mathrm{g}_{\mathrm{m} 1}}}
$$


$\mathrm{g}_{\mathrm{m} 3}=\frac{1}{\frac{1}{\mathrm{~g}_{\mathrm{m} 1}}+\frac{1}{\mathrm{~g}_{\mathrm{m} 2} \mathrm{~h}_{\mathrm{fe} 1}}}=\frac{1}{\frac{1}{4}+\frac{1}{(0.25) 30}}=2.61$ mho, where

$0.1=\frac{6+\frac{28}{1600}(7+0.38) 15}{1+g_{m 3} \frac{(1500)(15)}{28}+\frac{7}{40}+\frac{1}{(40)(4)}}$

$0.1=\frac{7.94}{1.181+804 \mathrm{~g}_{\mathrm{m} 3}} \quad \mathrm{~g}_{\mathrm{m} 3(\min )}^{\mathrm{c}}=\frac{7.82}{80.4}=0.097 \mathrm{mho}$

where $\mathrm{g}_{\mathrm{m} 3}^{\mathrm{c}}$ is the calculated value (minimum value desired) of mutual conductance.

10. A third Motorola 2N3946 can be used as the amplifier component; at this current level $(\sim 14$ mAdc):

$$
\mathrm{g}_{\mathrm{m} 3}^{\mathrm{D}} \cong 0.15 \quad \mathrm{~h}_{\mathrm{FE}} \mathrm{min}_{\min } \cong 40
$$

where $\mathrm{g}_{\mathrm{m} 3}^{\mathrm{D}}$ is the mutual conductance of the selected transistor and obtained using Ref. 3.

Since $\mathrm{g}_{\mathrm{m} 3}^{\mathrm{D}}>\mathrm{g}_{\mathrm{m} 3}^{\mathrm{C}}$ only one amplifier stage is needed for this case 11. Select $R_{2}$ and $R_{3}$ as follows:

Choose $\mathrm{I}_{3}=10$ mAdc (typically $\mathrm{I}_{3}<1 \% \mathrm{I}_{\mathrm{o}}$ to minimize power dissipation).

$$
\begin{aligned}
& \mathrm{V}_{3} \simeq \mathrm{V}_{\mathrm{R}}=15.0 \mathrm{Vdc} \\
& \mathrm{R}_{3}=\frac{\mathrm{V}_{3}}{\mathrm{I}_{3}}=\frac{15}{10 \times 10^{-3}}=1500 \text { ohms neglect } \mathrm{I}_{\mathrm{R}}
\end{aligned}
$$




$$
R_{2}=\frac{V_{o}-V_{3}}{I_{3}}=\frac{28-15}{10 \times 10^{-3}}=1300 \text { ohms }
$$

Use $1300 \mathrm{ohms}$ (the nearest standard value, $1200 \mathrm{ohms}$ would result in a $4 \%$ error in $V_{0}$ ).

The complete regulator circuit is shown in figure 4.

12. Efficiency considerations;

$$
\begin{aligned}
& \eta_{\text {rated }}=\frac{\mathbf{P}_{\mathrm{O}}}{\mathbf{P}_{\mathrm{O}}+\mathbf{P}_{\mathrm{L}}} \times 10^{2}=\frac{19.6}{38.9} \times 10^{2} \cong 50.3 \% \text { where } \\
& \mathbf{P}_{\mathrm{O}}=(0.70)(28)=19.6 \mathrm{~W} \\
& \mathbf{P}_{\mathrm{L}}=(0.70)(4.9+22.3)+(0.011)(28)=19+0.3=19.3 \mathrm{~W}
\end{aligned}
$$

\section{SHUNT CONVERTER DESIGN TECHNIQUE}

A step-by-step technique for the design of shunt converters is explained in this section. Equations used in this example are derived in Ref. 2. Referring to the block diagram in figure 2.

1. Conditions and requirements similar to those used for the series example:

$$
\begin{array}{ll}
\mathrm{V}_{\mathrm{O}}=+28 \mathrm{Vdc} & \Delta \mathrm{V}_{\mathrm{O}}= \pm 0.0125 \mathrm{Vdc} \\
\mathrm{V}_{\mathrm{S}}=+56 \mathrm{Vdc} & \Delta \mathrm{V}_{\mathrm{S}}= \pm 6 \mathrm{Vdc} \\
\mathrm{R}_{\mathrm{O}}=40 \mathrm{ohms} & \Delta \mathrm{R}_{\mathrm{O}}= \pm 15 \mathrm{ohms} \\
\mathrm{R}_{\mathrm{G}}=7 \mathrm{ohms} & \\
\text { Spacecraft baseplate temperature }-55^{\circ} \text { to }+60^{\circ} \mathrm{C}
\end{array}
$$


2. Select a shunt component:

$$
\begin{aligned}
& I_{\text {Sh }_{\text {max }}}=I_{o_{\max }}=1.12 \text { Adc where } I_{o_{\max }}=\frac{V_{o}+\Delta V_{o}}{R_{o}-\Delta R_{o}} \\
& =\frac{28.0125}{40-15}=1.12 \mathrm{~A}
\end{aligned}
$$

$I_{\mathrm{Sh}_{\max }}$ is defined as the limiting case which exceeds the maximum value of $I_{C E}$ obtained with a load equal to $R_{0}-\Delta R_{O}$

$\mathrm{V}_{\text {sh }} \leq \mathrm{V}_{\mathrm{o}}+\Delta \mathrm{V}_{\mathrm{o}} \simeq 28 \mathrm{Vdc}$ (forward biased steady state)

$\mathrm{PD}_{\mathrm{Sh}_{\max }}=\mathrm{I}_{\mathrm{sh}_{\max }} \mathrm{V}_{\mathrm{sh}_{\max }}=(28)(1.12)=31.4 \mathrm{~W}$ at $75^{\circ} \mathrm{C}$. Consider

Motorola type (Ref. 3) MJE205 2N4921

$\mathrm{BV}_{\mathrm{CEO}}$

$\mathrm{I}_{\mathrm{max}}$

$\mathrm{h}_{\mathrm{FE}}$ at $1.0,0.5 \mathrm{Adc}$

$50 \quad 40 \mathrm{Vdc}$

$\mathrm{V}_{\mathrm{CE}}$ at $1.5,0.5 \mathrm{Adc}$

$5.03 .0 \mathrm{Adc}$

PD at $75^{\circ} \mathrm{C}$

$50 \quad 45$

Cost

$0.51 .0 \mathrm{Vdc}$

$38 \quad 21.6 \mathrm{~W}$

$2.10 \quad 1.33 \$$

Choose the MJE205

3. Select the base-to-emitter resistor for the shunt component:

Let $\mathrm{I}_{2}=1.5 \mathrm{mAdc}$, typically $\mathrm{I}_{2} \leq 1 \% \mathrm{I}_{\mathrm{O}}=11.2 \mathrm{ma}$

$\mathrm{V}_{\mathrm{BE}_{1}}=0.8 \quad \mathrm{~V}_{\mathrm{BE}_{2}}=0.7 \mathrm{Vdc}$ (on bias two stages)

$R_{2}=\frac{V_{2}}{I_{2}}=\frac{1.5}{1.5 \times 10^{-3}}=1000 \mathrm{ohms}$

neglecting $\mathrm{I}_{3}<<\mathrm{I}_{2}$ 
4. Select the number of stages required in the shunt component:

Total $\mathrm{B}=\frac{1.12 \mathrm{~A}}{0.001}=1120$

Number of stages $\left(h_{F E} \cong 50\right.$ for each stage $)=50^{n} \geq 1120$

$\mathrm{n}=\mathbf{2}$

Choose a two stage series component.

5. For the second stage Q2:

$$
\begin{aligned}
& \mathrm{h}_{\mathrm{FE}_{2}}=\frac{\mathrm{h}_{\mathrm{FE}}}{\mathrm{h}_{\mathrm{FE}}}=\frac{1562}{1}=39.1 \\
& \mathrm{I}_{\mathrm{C} 2}=\frac{\mathrm{I}_{1}}{\mathrm{~h}_{\mathrm{FE}}}=\frac{0.70}{50}=14 \mathrm{mAdc}
\end{aligned}
$$

$\begin{array}{lrr}\text { Motorola type (Ref. 3) } & 2 \mathrm{~N} 3946 & 2 \mathrm{~N} 3947 \\ \mathrm{BV}_{\mathrm{CEO}} & 40 & 40 \mathrm{Vdc} \\ \mathrm{I}_{\mathrm{O} \text { max }} & 200 & 200 \mathrm{mAdc} \\ \mathrm{PD}_{\mathrm{T}} \text { at } 75^{\circ} \mathrm{C} & 0.96 & 0.96 \mathrm{~W} \\ { }_{\mathrm{h}} \text { at } 14 \mathrm{mAdc} & 100 & 150 \\ \ddot{C o s t}_{\text {Cost }} & 2.40 & 2.75 \$\end{array}$

Choose the 2N3946

6. Select a reference component (RC)

Let $R_{\mathrm{f}} \leq 5$ ohms

$$
\begin{aligned}
& \mathrm{V}_{\mathrm{R}}=\mathrm{V}_{\mathrm{o}}-\mathrm{V}_{2}=28-1.5=26.5 \mathrm{Vdc} \\
& \mathrm{I}_{1_{\max }}=\mathrm{I}_{2}+\mathrm{I}_{3}=1.5+0.14=1.64 \mathrm{mAdc} \\
& \mathrm{PD}_{\mathrm{RC}}=26.5(1.64)=43.5 \mathrm{~mW} \text { at } 75^{\circ} \mathrm{C} \text {. Consider }
\end{aligned}
$$


Motorola type (Ref. 3)

IN 4750

IN971

Selected zener voltage $26.5 \pm 1.0 \%$

$26.5 \pm 1.0 \% \mathrm{Vdc}$

$\mathrm{PD}_{\mathrm{z}}$ at $75^{\circ} \mathrm{C}$

0.93

$0.35 \mathrm{~W}$

$\mathrm{I}_{\mathrm{R}}$

34

15 mAdc

$\mathrm{z}_{\mathrm{zt}}$ at $9.5 \mathrm{mAdc}$

35

41 ohms

Cost

1.26

$2.35 \$$

Choose the IN4750 (selected)

7. Select a series resistor component:

$$
\begin{aligned}
& V_{S}-\Delta V_{S}=V_{O}+\left(R_{G}+R_{1}\right) I_{G} \\
& 56-6=28+\left(R_{G}+R_{1}\right) 0.70 \\
& 22=\left(R_{G}+R_{1}\right) 0.70 \\
& R_{1}=31.5-7=24.5 \text { ohms }
\end{aligned}
$$

Choose $24 \mathrm{ohms}$ (closest standard value).

The complete circuit for the transistorized shunt voltage converter is shown in figure 5 .

8. Efficiency considerations:

$$
\begin{aligned}
\eta= & \frac{P_{O}}{P_{O}+P_{L}} \times 10^{2}=\frac{19.6}{19.6+29.5} \times 10^{2}=38.9 \\
P_{O} & =V_{O} I_{O}=(28)(0.70)=19.6 \mathrm{~W} \\
P_{L} & =I_{G}\left(R_{G}+R_{1}\right)+\left(I_{S h}+I_{C 2}+I_{1}\right) V_{O} \\
& =(0.70)(31.5)+(0.266)(28)=29.5 \mathrm{~W}
\end{aligned}
$$




\section{SWITCHING CONVERTER DESIGN TECHNIQUE}

A step-by-step technique for the design of switching converters is explained in this section. Equations used in this example are derived in Refs. 4 and 5. The block diagram of a switching converter is illustrated in figure 3 .

1. Conditions and requirements similar to those used for the series example:

$\mathrm{V}_{\mathrm{O}}=+28 \mathrm{Vdc} \quad \Delta \mathrm{V}_{\mathrm{O}}= \pm 0.030 \mathrm{Vdc}$

$\mathrm{V}_{\mathrm{S}}=+56 \mathrm{Vdc} \quad \Delta \mathrm{V}_{\mathrm{S}}= \pm 6 \mathrm{Vdc}$

$R_{\mathrm{O}}=40 \mathrm{ohms} \cdot \Delta \mathrm{R}_{\mathrm{o}}= \pm 15 \mathrm{ohms}$

$\mathrm{R}_{\mathrm{G}}=7$ ohms

Spacecraft baseplate temperature $-55^{\circ}$ to $+60^{\circ} \mathrm{C}$

2. Select a choke for the output filter using energy considerations with Kirchoff's laws:

$$
L_{F}=\frac{\left(V_{S}-V_{o}\right) V_{O}}{2 f_{S}\left(\Delta I_{o}\right)}=\frac{(56-28)(28)}{(2)\left(2.5 \times 10^{4}\right)(56)(0.25)}=1.12 \mathrm{mH} \text {, where } f \text { is }
$$

the desired frequency at maximum load

An air gaped ' $\mathrm{C}$ ' core would be best suited for the choke component

3. Select a capacitor for the output filter:

$$
\begin{aligned}
& \mathrm{C}_{\mathrm{F}}=\frac{\left(\mathrm{V}_{\mathrm{S}}-\mathrm{V}_{\mathrm{o}}\right) \mathrm{V}_{\mathrm{O}}}{8 \mathrm{f}^{2}\left(\Delta \mathrm{V}_{\mathrm{o}}-\Delta \mathrm{H}\right) \mathrm{L}_{\mathrm{F}} \mathrm{V}_{\mathrm{S}}}=\frac{(56-28)(28)}{8\left(2.5 \times 10^{4}\right)^{2}\left(6 \times 10^{-2}\right)\left(1.12 \times 10^{-3}\right)(56)} \\
& \mathrm{C}_{\mathrm{F}}=29.5 \mu \mathrm{F} \text { where } \Delta \mathrm{H} \text { is the hysteresis of the voltage compar- } \\
& \quad \begin{array}{l}
\text { ator which in the case for a differential input amplifier is as- } \\
\text { sumed to be zero. }
\end{array}
\end{aligned}
$$

Use $2,15 \mu \mathrm{F} \pm 10 \%, 50$ WVDC aluminum electrolytes in parallel to minimize equivalent series resistance 
4. Select a transistor for the series switching component:

$\mathrm{I}_{\mathrm{C}_{\max }} \geq 1.0 \mathrm{Adc}$

Assuming a converter efficiency of $80 \%$, the following relationship for transistor power dissipation is:

\begin{tabular}{|c|c|c|c|}
\hline \multicolumn{4}{|c|}{$\begin{array}{l}\mathrm{PD}_{\mathrm{T}}-0.2 \mathrm{P}_{\mathrm{O}}=(0.2)(28)(0.75)=4.2 \mathrm{~W} \text { at } 75^{\circ} \\
\mathrm{V}_{\mathrm{CEO}} \geq 62 \mathrm{Vdc} \quad \mathrm{V}_{\mathrm{CE}} \leq 0.3 \mathrm{Vdc}\end{array}$} \\
\hline Motorola type (Ref. 3) & $2 \mathrm{~N} 4912$ & $2 \mathrm{~N} 4923$ & \\
\hline $\mathrm{I}_{\mathrm{C}_{\max }}$ at $\mathrm{I}_{\mathrm{B}}=0.1 \mathrm{Adc}$ & 1.0 & 1. 0 Adc & \\
\hline $\mathrm{PD}_{\mathrm{T}}$ at $75^{\circ} \mathrm{C}$ & 20 & $21 \mathrm{~W}$ & \\
\hline $\mathrm{V}_{\mathrm{CEO}}$ & 80 & $80 \mathrm{Vdc}$ & \\
\hline $\mathrm{V}_{\mathrm{CES}}$ & 0.3 & $0.3 \mathrm{Vdc}$ & \\
\hline$f_{T}$ & 3.0 & $3.0 \mathrm{MHz}$ & \\
\hline $\mathrm{t}_{\mathrm{T} \text { (on/off switching time) }}$ & 0.3 & $0.3 \mu \mathrm{sec}$ & \\
\hline Cost & 2.10 & $1.80 \$$ & \\
\hline
\end{tabular}

Use the $2 \mathrm{~N} 4923$.

5. Select a diode for the output filter (Ref. 7):

$\begin{array}{lrr}\mathrm{I}_{\mathrm{D}_{\max }=1.0 \mathrm{Adc}} & \text { PIV }_{\mathrm{D}} \geq 28 \mathrm{Vdc} & \\ \mathrm{t}_{\mathrm{fr}} \leq 0.2 \mu \mathrm{sec} & \mathrm{t}_{\mathrm{rr}} \leq 0.3 \mu \mathrm{sec} . & \text { Consider } \\ \text { Unitrode type (Ref. } 6) & \text { UTR } 02 & \text { UTX 205 } \\ \mathrm{I}_{\mathrm{D}_{\max }} \text { at } 75^{\circ} \mathrm{C} & 1.54 & 1.54 \mathrm{Adc} \\ \text { PIV }_{\mathrm{D}} & 50.0 & 50.0 \mathrm{~V} \\ \mathrm{t}_{\mathrm{fr}} & 0.1 & 0.01 \mu \mathrm{sec} \\ \mathrm{t}_{\text {rr }} & 0.25 & 0.075 \mu \mathrm{sec} \\ \text { Cost } & 1.55 & 3.55 \$\end{array}$

Use UTR 02. 
6. Efficiency considerations.

$$
\begin{aligned}
& \eta=\frac{\mathrm{P}_{O}}{\mathrm{P}_{\mathrm{O}}+\mathrm{P}_{\mathrm{L}}} \times 10^{2}=\frac{19.6}{19.6+2.9} \times 10^{2}=87.2 \% \\
& \mathrm{P}_{\mathrm{O}}=(28)(0.70)=19.6
\end{aligned}
$$

Losses:

a. Choke dc loss $=I_{0}^{2} R_{C H}=(1.12)^{2}(1)=1.26 \mathrm{~W}$

b. Choke ac loss $=\left(\frac{\mathrm{f}^{2} \mathrm{~L}^{2}}{6 \mathrm{Q}}\right)\left[\frac{\mathrm{V}_{\mathrm{s}} \mathrm{I}_{\mathrm{max}}^{3}-\mathrm{I}_{\mathrm{o}}^{3}}{\mathrm{~V}_{\mathrm{o}}\left(\mathrm{V}_{\mathrm{s}}-\mathrm{V}_{\mathrm{o}}\right)}\right]$

$$
\begin{aligned}
& =\left[\frac{\left(2.5 \times 10^{4}\right)^{2}\left(1.12 \times 10^{-3}\right)^{2}}{(6)(20)}\right]\left\{\frac{56\left[(1)^{3}-(0.70)^{3}\right]}{28(56-28)}\right\} \\
& =0.216 \mathrm{~W}
\end{aligned}
$$

c. Transistor on loss $\cong \frac{V_{\text {CEsat }} V_{0} I_{0}}{V_{S}}=\frac{(0.3)(28)(0.70)}{(56)}$

$$
=0.104 \mathrm{~W}
$$

d. Diode on loss $\cong \mathrm{V}_{\mathrm{fr}} \mathrm{I}_{\mathrm{o}} \frac{\left(\mathrm{V}_{\mathrm{s}}-\mathrm{V}_{\mathrm{o}}\right)}{\mathrm{V}_{\mathrm{S}}}=\frac{(0.6)(0.70)(28)}{56}$

$$
=0.21 \mathrm{~W}
$$


e. Transistor switching losses:

$$
\begin{aligned}
& \mathrm{fI}_{\mathbf{P}} \mathrm{V}_{\mathrm{s}} \mathrm{t}_{\mathrm{rr}(\text { off })}+\frac{\mathrm{fV}_{\mathrm{S}} \mathrm{I}_{\max }\left[\mathrm{t}_{\mathrm{T} \text { (off) }}^{2}+\mathrm{t}_{\mathrm{rr} \text { (off })}^{2}\right]}{6 \mathrm{t}_{\mathrm{T} \text { (off) }}}=(3)(56)\left(0.25 \times 10^{-6}\right)\left(2.5 \times 10^{4}\right) \\
& +\left(2.5 \times 10^{4}\right)(56)(1)\left(0.09 \times 10^{-12}+0.06 \times 10^{-12}\right) \\
& \text { (6) }\left(0.3 \times 10^{-6}\right) \\
& =1.05+0.0128=1.063 \mathrm{~W} \\
& \text { f. Auxiliary circuit losses } \cong \frac{\mathrm{V}_{\mathrm{B}} \mathrm{I}_{\mathrm{O}} \mathrm{V}_{\mathrm{o}}}{\mathrm{h}_{\mathrm{FE}} \mathrm{V}_{\mathrm{S}}}=\frac{(5)(1.12)(28)}{(10)(56)} \\
& =0.28 \mathrm{~W} \\
& \text { Total losses }=\sum_{i=a}^{f} P_{i} \cong 3.13 \mathrm{~W}
\end{aligned}
$$

The complete schematic at the switching converter is shown in figure 6. The design techniques for the auxiliary circuit components are explained in existing literature (see Ref. 8). The important requirements for these circuits are that hysteresis be held to a minimum and rise and fall times are less than the series component.

A study of optimum methods to transfer and transform electric power in aerospace applications showed that no single method was best for all cases. The optimum method was very much dependent upon available devices, generating sources, and load parameters. These are constraints difficult to identify for future aerospace missions.

Table I shows some of the important typical converter parameters to consider when faced with the task of choosing the type of converter for use in a given mission.

Low efficiency increases power source area and raises costs; i.e., solar arrays cost about $\$ 8000$ per square foot at about 4 watts per square foot. It is not unusual to spend $\$ 10^{6}$ for a 500 watt, 125 square foot array. 


\section{CONCLUDING REMARKS}

The design of transistorized voltage converters of the series, shunt, and switching types are developed and explained in this paper.

Past aerospace missions have used either the series, shunt and switching converters or combinations thereof to transfer and transform electric power. The shunt converter has the smallest size, lowest weight and parts count. Regulation and stability are very good but efficiency is poor. The series converter is somewhat larger in size, heavier, uses more parts and has an order of magnitude (10 to 1) decrease in regulation performance than the shunt converter. Stability of both types are good at light or full loads. A ten percent increase in efficiency justifies its use in some cases.

Many current aerospace missions are going to some form of the switching converter. It tends to be a compromise with increased size, weight, and circuit complexity to gain in efficiency and regulation over a series converter. A switching converter will usually exhibit ringing in the output filter for some types of loads so it has only fair stability performance.

The best designed product is only as good as the people and materials finally used to make it. The task of determining that people with the required skills and materials of the proper specified quality are used to build a product must be included in power processor design by other means. This task requires participation of many disciplines including rigorous reliability and quality assurance engineering (Ref. 9). 


\section{RE FERENCES}

1. Schwarz, F. C.: Power Processing. NASA SP-244, 52, 1971.

2. Anon.: Transistorized Voltage Regulators. AG ICE-254, RCA, Somerville, New Jersey, 12, 1961.

3. Anon.: The Semiconductor Data Book. RRD 97084, Motorola, Phoenix, Arizona, 450, 1970 .

4. Hauser, J. A.: Get With Switching Voltage Regulators. Electronic Design, 62-66, April., 1968.

5. Schoenfeld, A. A.: The Application of the Analog Signal to Discrete Time Interval Converter to the Signal Conditioner Power Supplies, TRW Inc., TRW Systems Group; NASA CR-120906, 89 (1972).

6. Anon.: Design Guide and Short Form Catalog. Catalog SF-1A, Unitrode Corp., Watertown, Mass., 32, (1971).

7. Eby, Ed: Choose the Right Commutating Diode for Switching Regulators. EDN/EEE, 22-24 (1971).

8. Millman, J. and Taub, H.: Pulse, Digital, and Switching Waveforms. McGraw-Hill, 1965.

9. Lalli, V. R。; and Vargo, D. J。: Aerospace Reliability Applied to Biomedicine. NASA TM X-67942 (1971). 
TABLE I. - TYPICAL CONVERTER COMPARISON

\begin{tabular}{|l|c|c|c|c|c|c|}
\hline Type & $\begin{array}{r}\text { Efficiency, } \\
\text { percent }\end{array}$ & Size & Weight & $\begin{array}{c}\text { Parts } \\
\text { count }\end{array}$ & Regulation & Stability \\
\hline Series & 50 & 1.1 & 1.2 & 7 & \pm 0.10 & Good \\
Shunt & 40 & 1.0 & 1.0 & 5 & \pm 0.0125 & Good \\
Switching & 85 & 1.3 & 1.6 & 24 & \pm 0.03 & Fair \\
\hline
\end{tabular}




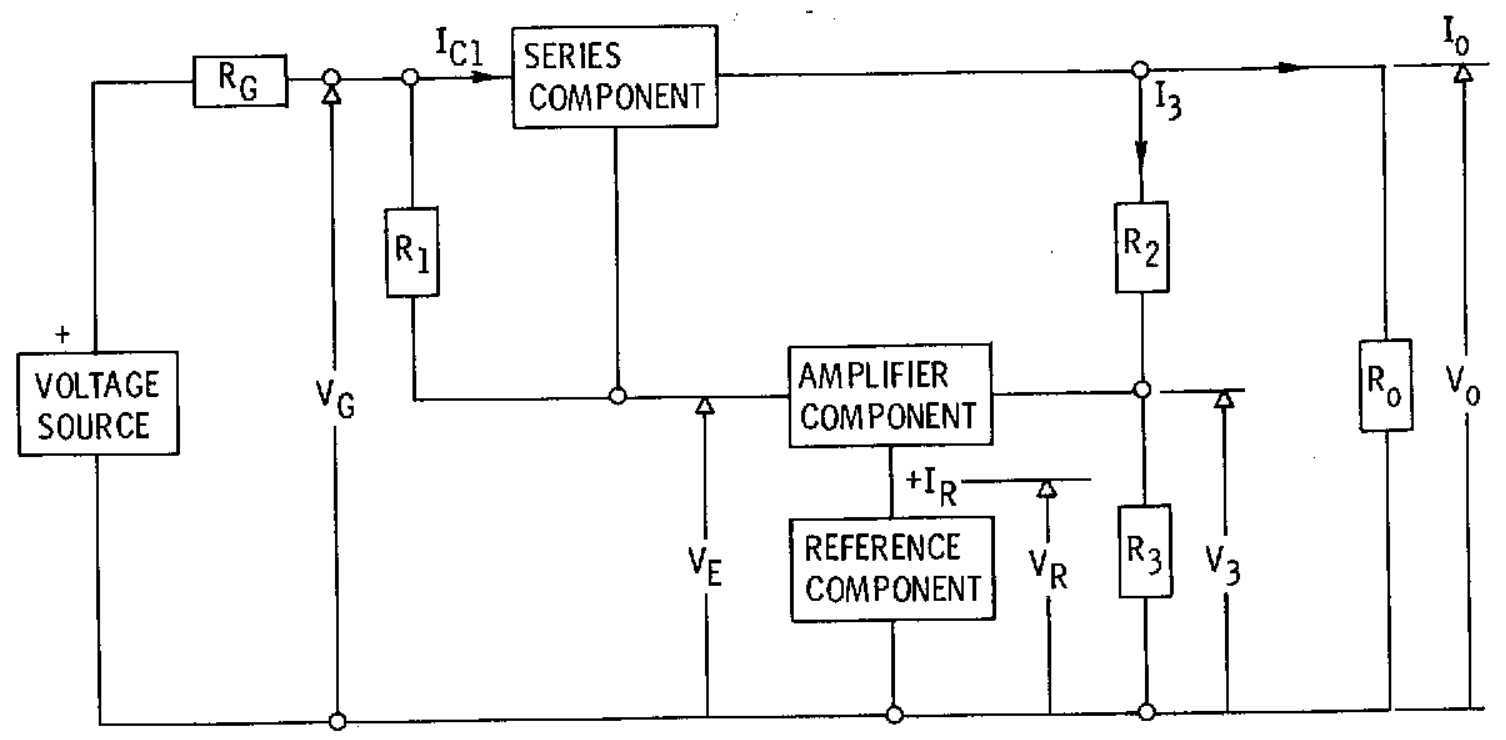

Figure 1. - Typical series voltage converter.

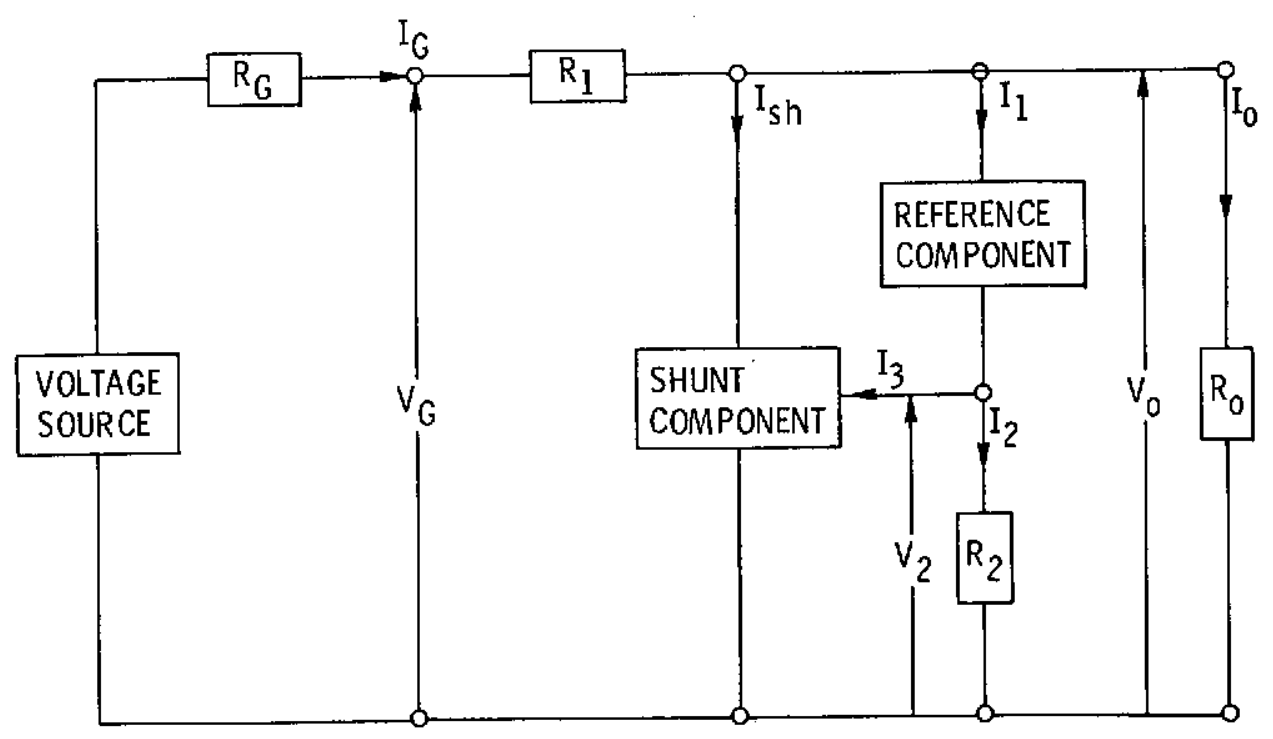

Figure 2. - Typical shunt voltage converter. 


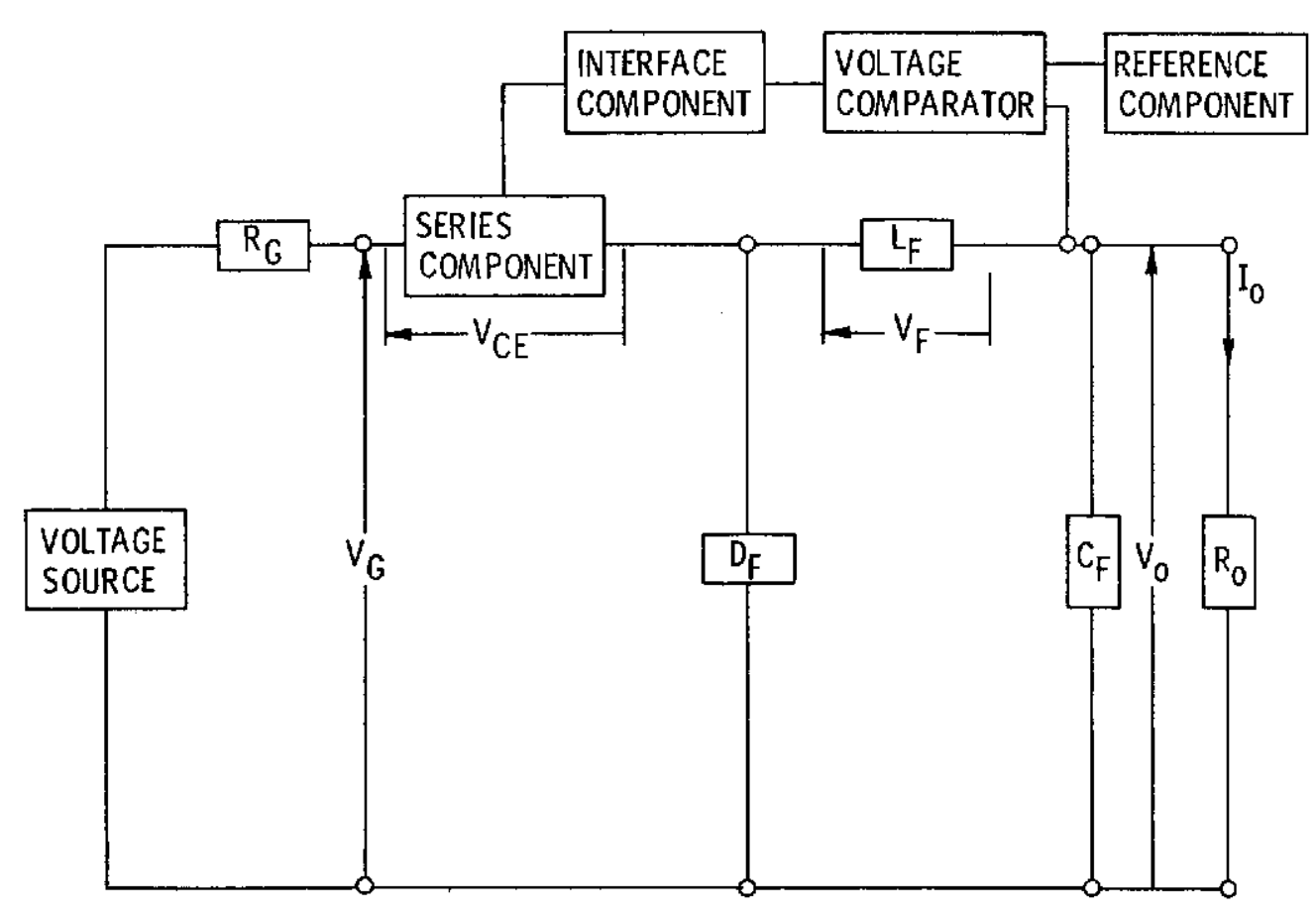

Figure 3. - Typical switching voltage converter.

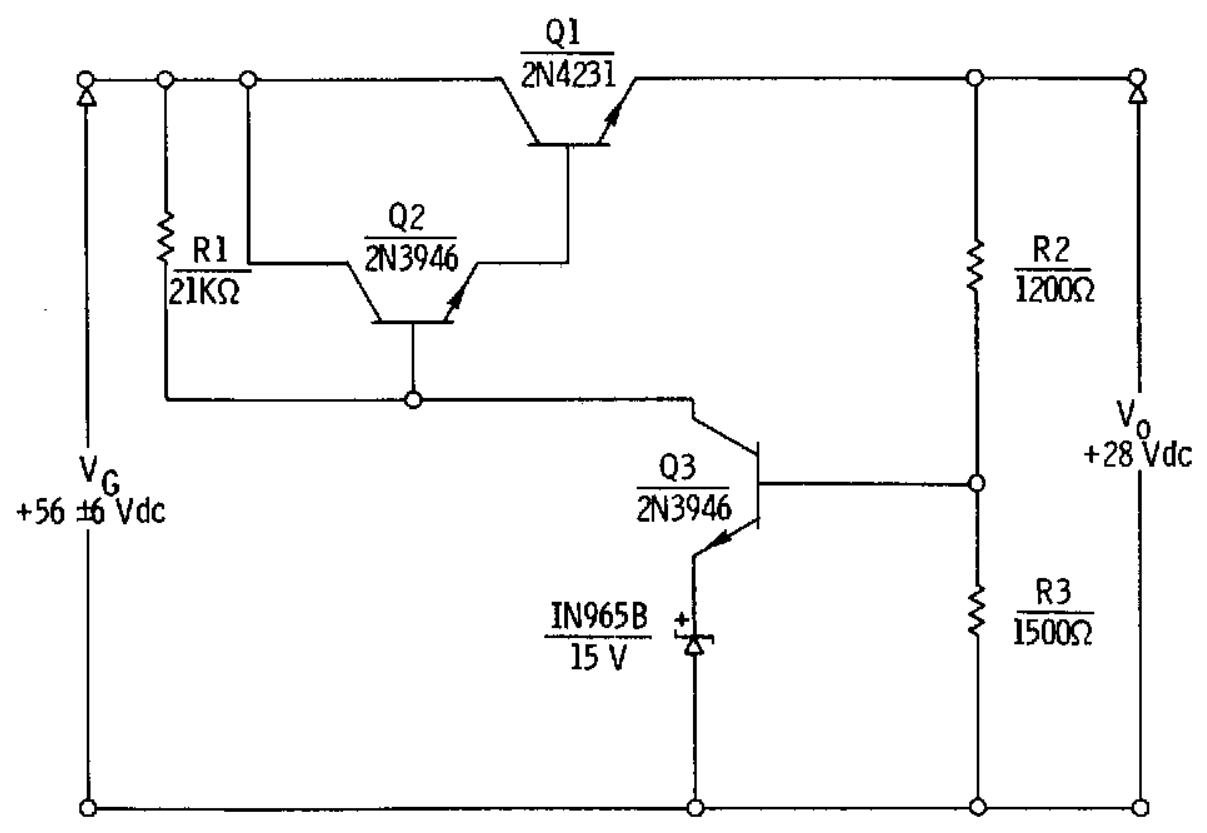

Figure 4. - Schematic diagram for $28 \mathrm{~V}$ series converter. 


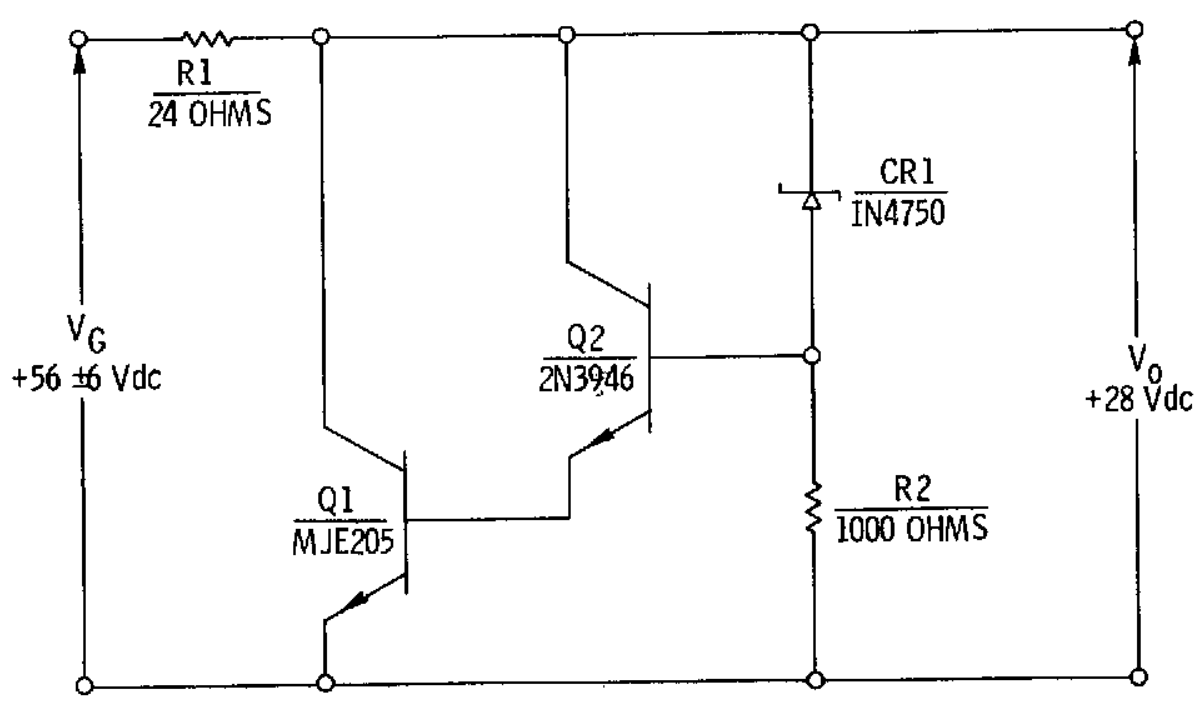

Figure 5. - Schematic diagram for $28 \mathrm{~V}$ shunt converter.

2
5
5
1
1

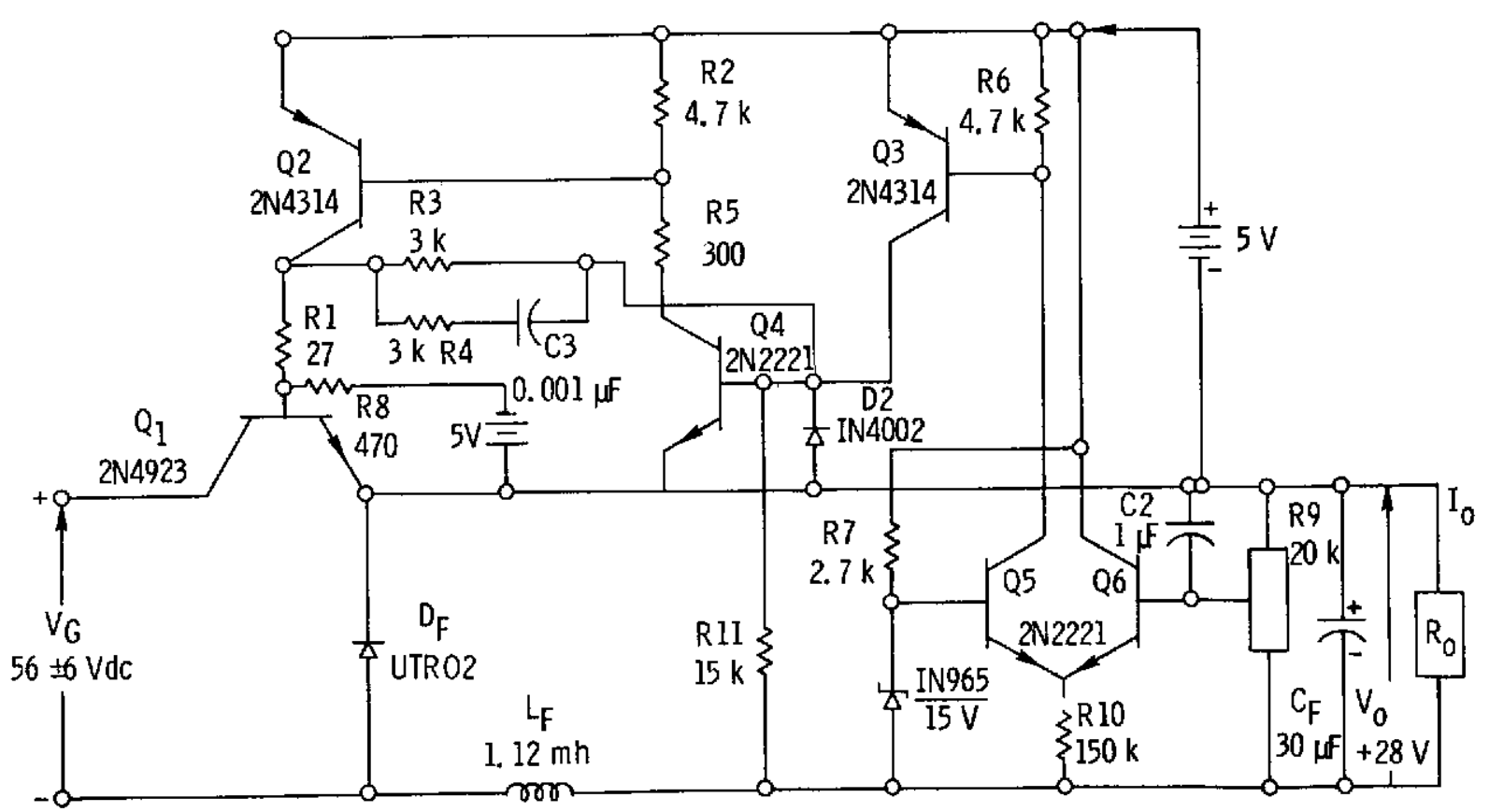

Figure 6. - Schematic diagram for $28 \mathrm{~V}$ switching converter. 